\title{
IDENTIFYING AND IMPOSING PARTIAL EQUILIBRIUM IN CHEMICALLY REACTING SYSTEMS
}

\author{
David R. Mott* and Elaine S. Oran ${ }^{\dagger}$, \\ Laboratory for Computational Physics and Fluid Dynamics \\ Naval Research Laboratory, Washington, D.C. 20375 \\ and \\ Bram van Leer ${ }^{\ddagger}$ \\ The University of Michigan \\ Ann Arbor, Michigan 48109-0578
}

\begin{abstract}
We develop and describe a process for identifying and imposing partial equilibrium (PE) in systems of coupled ordinary differential equations representing reaction kinetics, with particular relevance to reacting flow applications. First, a method for identifying equilibrium reactions is developed. Then, a systematic way of identifying the conserved scalars and linearly independent equilibrium constraints is described. The traditional algebraic constraint used for partial equilibrium is then modified, which improves the transition when a reaction first reaches equilibrium. The PE tools are demonstrated with the kinetic integrator CHEMEQ2, and the results illustrate that the new methods allow equilibrium to be imposed sooner than with more conventional approaches, while still maintaining solution accuracy.
\end{abstract}

\footnotetext{
${ }^{*}$ Senior Member, AIAA

${ }^{\dagger}$ Fellow, AIAA

${ }^{\ddagger}$ Department of Aerospace Engineering. Fellow, AIAA
}

\section{Introduction}

Many applications in science and engineering involve coupled sets of dependent variables $\left\{y_{i}\right\}$ and their rates of change, $\left\{g_{i}\right\}$. These relationships are represented mathematically by systems of coupled, nonlinear ordinary differential equations (ODEs) of the form

$$
\frac{d y_{i}}{d t}=g_{i}, \quad 1 \leq i \leq n
$$

Coupled sets of chemical reactions can be written in this form for the species concentrations $\left\{y_{i}\right\}$, with each $g_{i}$ being a linear combination of the source terms for individual reactions in the chemical mechanism. Often the timescales for the various species differ by many orders of magnitude, and there is strong coupling between species. In this case, the set of equations represented by Eq. (1) is considered stiff and is not readily solved by classical methods, such as the low-order Euler methods or higher-order Adams-Moulton methods. ${ }^{1-3}$

Partial-equilibrium (PE) methods overcome stiffness by altering the source terms used in the ODE integration. ${ }^{4}$ For example, consider sets of coupled equations representing chemical reactions. If the effects of fast reactions can be calculated using algebraic equilibrium constraints, these reactions may be removed from the standard kinetic source term calculation. The effects of these reactions are included either by (a) directly imposing the algebraic constraints, ${ }^{5-8}$ or (b) differentiating these constraints

American Institute of Aeronautics and Astronautics 
to obtain a less-stiff form of the source term..$^{5,9,10}$ In this paper, we develop a method for approach (a), and we compare this new method to several developed previously. ${ }^{5,10}$

For the equilibrium constraint to be accurate, the reaction must be fast compared to the timestep of the integration, and it should also be close to equilibrium by some measure. Although the examples presented in Section 7 are limited to single-point integration problems, the particular difficulties associated with identifying and imposing equilibrium in reacting-flow problems are our focus. Using a process-split reacting flow model, the effects of each process such as advection and diffusion are calculated separately during a timestep. Therefore, identifying proximity to equilibrium is particularly difficult because equilibrium imposed at the end of the preceding chemical timestep can be disturbed by the other processes that alter the species concentrations. This means that the equilibrium reactions must compensate for changes caused by these other processes that disturb their equilibrium.

PE methods exploit the same system characteristics as Computational Singular Perturbation (CSP $)^{11-14}$ and the method of Intrinsic LowDimensional Manifolds (ILDM). ${ }^{15-17}$ All three approaches use the fastest modes in the system to establish algebraic relationships that can be used to make the chemistry calculation less costly. As with CSP and ILDM, PE methods can be used to reduce the number of ODEs that are integrated, but we do not take this approach. Here, we use partial equilibrium to reduce the stiffness of the ODE system, not to reduce the size of the ODE system.

We present a new method for determining if equilibrium can be assumed for a given reaction. This test includes the calculation of a single timescale for the reaction rather than separate timescales for each of the species in the reaction, as well as an estimate of how close the reaction will be to equilibrium at the end of the timestep point. We also present a procedure for identifying redundant equilibrium constraints, and this procedure provides a set of conserved scalars for an arbitrary reaction set. The classic partial equilibrium constraint (i.e., forcing the source terms of equilibrium reactions to be identically zero) is now replaced. The new constraint recognizes that the equilibrium reactions must con- tinually compensate for other processes present in the system that disturb the equilibrium, such as the slower chemical reactions, or advection and diffusion in a reacting-flow problem. Therefore, a non-zero source term for the equilibrium reactions is imposed that is consistent with the reaction's efforts to maintain equilibrium. This new constraint allows equilibrium to be imposed sooner in a simulation while maintaining the accuracy of the integration.

\section{Background}

Here we introduce concepts and definitions needed to develop the methods in the following sections.

A progress variable $\lambda$ is a scalar that provides a measure of how far a reaction has taken the concentrations from their original values. ${ }^{18}$ Consider the simple reversible reaction (i.e., a pair of forward and backward reactions) for species $A$ and $B$

$$
A \rightleftarrows 2 B
$$

with source term

$$
F=k_{f} y_{A}-k_{b} y_{B}^{2},
$$

for concentrations $y_{A}$ and $y_{B}$ and forward and backward rate constants $k_{f}$ and $k_{b}$, respectively. The loss of one molecule of species $A$ produces two molecules of $B$, so for changes $\Delta y_{A}$ and $\Delta y_{B}$ in the concentrations of $A$ and $B$,

$$
\Delta y_{B}=-2 \Delta y_{A}
$$

Consider $\lambda$ which satisfies

$$
\frac{d \lambda}{d t}=F
$$

We will refer to the $\lambda$ that obeys Eq. (5) and has initial value $\lambda^{0}=0$ as the natural progress variable. This choice for $\lambda$ is convenient because multiplying $\lambda$ by the stoichiometric coefficient of a given species, with a minus sign for reactants and a plus sign for products (as determined by the sign given to $F$ ), gives the perturbation for that species:

$$
\Delta y_{A}=-\lambda, \Delta y_{B}=2 \lambda
$$


Therefore replacing $y_{A}$ and $y_{B}$ in Eq. (3) with

$$
\begin{aligned}
& y_{A}=y_{A}^{0}-\lambda \\
& y_{B}=y_{B}^{0}+2 \lambda
\end{aligned}
$$

allows $F$ to be written in terms of $\lambda$ and the initial concentrations, $y_{A}^{0}$ and $y_{B}^{0}$, at the start of the timestep. Therefore Eq. (5) is a single equation in a single unknown, $\lambda$. The value of $\lambda$ at any time $t$ gives the concentration values through Eqs. (7) and (8).

To generalize these concepts to an arbitrary number or reactions and concentrations, we view the species number densities as components of a composition vector, $\mathbf{y}: \mathbf{y}=\left(y_{1}, y_{2}, \ldots, y_{n}\right)$. The vector $\mathbf{y}$ lies in an $n$-dimensional vector space $\Phi$ for which a vector $\mathbf{y} \in \Phi$ gives a specific composition; component $y_{i}$ of $\mathbf{y}$ in the natural coordinates gives the number density of species $A_{i}$. Any reaction involving these $n$ species can be written in the form

$$
\sum_{i=1}^{n} a_{i} A_{i} \rightleftarrows \sum_{i=1}^{n} b_{i} A_{i}
$$

for some scalars $\left\{a_{i}\right\}$ and $\left\{b_{i}\right\}$. For the reaction in Eq. (2), assigning $A_{1}$ to $A$ and $A_{2}$ to $B$ gives $a_{1}=1$, $b_{2}=2$, and $a_{i}=0, b_{i}=0$ otherwise.

The stoichiometry of the reaction defines a vector $\mathbf{r} \in \Phi$, which is given by

$$
\mathbf{r}=\left(b_{1}-a_{1}, b_{2}-a_{2}, \ldots, b_{n}-a_{n}\right) .
$$

The vector $\mathbf{r}$ defines a line in $\Phi$ (i.e., a onedimensional subspace of $\Phi$ ) along which the composition can move due to this reaction. Therefore, given an initial composition $\mathbf{y}^{0}=\left(y_{1}^{0}, y_{2}^{0}, \ldots, y_{n}^{0}\right) \in$ $\Phi$, any composition $\mathbf{y}$ achieved through the single reversible reaction in Eq. (9) must be of the form

$$
\mathbf{y}=\mathbf{y}^{0}+\lambda \mathbf{r}
$$

for some scalar $\lambda$. The progress variable can also be used to track a thermodynamics variable such as sensible energy by adding a component to $\mathbf{r}$. If a set of $k$ reactions occur, then the $j^{t h}$ reaction defines a vector $\mathbf{r}^{j}=\left(r_{1}^{j}, r_{2}^{j}, \ldots, r_{n}^{j}\right)$, and the final composition can be written as

$$
\mathbf{y}=\mathbf{y}^{0}+\sum_{j=1}^{k} \lambda_{j} \mathbf{r}^{j}
$$

for some scalars $\left\{\lambda_{j}\right\}$.

Finally, a conserved scalar is a linear combination of the species concentrations for which the chemical source term vanishes. Equations. (7) and (8) indicate that

$$
y_{B}+2 y_{A}=\text { constant }=y_{B}^{0}+2 y_{A}^{0},
$$

so this combination of concentrations acts as a conserved scalar for Eq. (2) acting alone. More generally, given a set of $k$ reaction vectors $\left\{\mathbf{r}^{j}\right\}$, any vector $\mathbf{c}=\left(c_{1}, c_{2}, \ldots, c_{n}\right) \in \Phi$ which is perpendicular to each of the $k$ vectors $\left\{\mathbf{r}^{j}\right\}$ defines a conserved scalar. Taking the dot product of $\mathbf{c}$ with $\mathbf{y}-\mathbf{y}^{0}$ yields

$$
\begin{aligned}
& \mathbf{c} \cdot\left(\mathbf{y}-\mathbf{y}^{0}\right)=\mathbf{c} \cdot \sum_{j=1}^{k} \lambda_{j} \mathbf{r}^{j}=0 \\
& \mathbf{c} \cdot \mathbf{y}=\mathbf{c} \cdot \mathbf{y}^{0}, \text { or } \\
& \sum_{j=1}^{n} c_{i} y_{i}=\sum_{j=1}^{n} c_{i} y_{i}^{0} .
\end{aligned}
$$

Equation (16) is a conservation law for the set of reactions $\left\{\mathbf{r}^{1}, \mathbf{r}^{2}, \ldots, \mathbf{r}^{k}\right\}$, which relates $\left\{y_{i}\right\}$ through a constant determined by the initial conditions.

Define $R \subset \Phi$ as the subspace of $\Phi$ spanned by the vectors $\mathbf{r}^{1}, \mathbf{r}^{2}, \ldots, \mathbf{r}^{k}$. The orthogonal complement $C$ of $R$ is the subspace spanned by all vectors $\mathbf{c}$ that define conserved scalars for the reactions in $R$. A basis $\left\{\mathbf{c}^{1}, \mathbf{c}^{2}, \ldots, \mathbf{c}^{m}\right\}$ for $C$ gives a complete set of conserved scalars of the form of Eq. (16) for the given reaction set. These subspaces and basis vectors can be readily constructed, as is illustrated in Section 5 .

\section{Traditional Approach for Identifying Equilibrium}

Partial-equilibrium methods inspect individual reactions in the mechanism, not the composite source terms $\left\{g_{i}\right\}$. Consider the reversible reaction given in Eq. (17) for species $A, B, C$, and $D$,

$$
A+B \rightleftarrows C+D \text {. }
$$

The source term, $F$, for this reaction is given by the law of mass action as

$$
F=k_{f} y_{A} y_{B}-k_{b} y_{C} y_{D},
$$


in which $k_{f}$ and $k_{b}$ are forward and backward rate constants that are functions of the thermodynamic state, and the subscript on each concentration variable $y$ indicates species.

Partial equilibrium is assumed for the reaction in Eq. (17) if the forward and backward rates are fast enough relative to the other processes to maintain $F \approx 0$. The source term $F$ will not stay identically zero as the system evolves because other processes change the concentrations and the rate constants. Rather than calculating $F$ and including it in the species source terms, the effect of the reaction is included by imposing some form of $F \approx 0$.

Classically, a fast reaction is identified by calculating separate timescales for each of the four species in the reaction. The ODE for species $A$, for this single reversible reaction, is

$$
\frac{d y_{A}}{d t}=q_{A}-\frac{y_{A}}{\tau_{A}}
$$

where $q_{A}$ and $\tau_{A}$ are

$$
q_{A}=k_{b} y_{C} y_{D}, \tau_{A}=\frac{1}{k_{f} y_{B}} .
$$

If $q_{A}$ and $\tau_{A}$ were constant, the solution for Eq. (19) would be

$$
y_{A}(t)=y_{A}^{0} e^{-t / \tau_{A}}+q_{A} \tau_{A}\left(1-e^{-t / \tau_{A}}\right),
$$

so $\tau_{A}$ acts as a timescale. Similar definitions give time constants for the other species as well:

$$
\tau_{B}=\frac{1}{k_{f} y_{A}}, \tau_{C}=\frac{1}{k_{b} y_{D}}, \tau_{D}=\frac{1}{k_{b} y_{C}} .
$$

By comparing the time constants to some specified threshold or, more appropriately, the chemical timestep used in the integration, the reactions can be classified as fast or slow. This comparison is usually done with the species that has the largest timescale to prevent the algebraic equilibrium constraint from being applied prematurely.

To determine if $F$ is sufficiently close to zero, the net rate $F$ may be compared to its forward and backward components:

$$
\frac{\|F\|}{k_{f} y_{A} y_{B}+k_{b} y_{C} y_{D}}<\epsilon_{\text {small }} .
$$

An alternate check would use the equilibrium values predicted by the simplified form in Eq. (21),

$$
\left(y_{A}\right)_{e q}=q_{A} \tau_{A}
$$

and similar expressions for $\left(y_{B}\right)_{e q},\left(y_{C}\right)_{e q}$, and $\left(y_{D}\right)_{e q}$. If all four species are within some tolerance of these equilibrium values and their timescales are sufficiently small, the reaction is assumed to be in equilibrium.

Although this process for identifying PE is widely used, it is unsettling that a single reversible reaction provides four separate measures of the speed of the reaction and its proximity to equilibrium. This ambiguity arises because the timescale calculation for one species assumes that the other three concentrations are constant, which is obviously not correct. Also, in a process-split reacting-flow solver, a constraint based on Eq. (23) or Eq. (24) may fail to identify equilibrium present in the system due to the concentration changes caused by other processes during the timestep.

\section{New Method for Identifying Equilibrium}

\subsection{Two-Body Reactions}

A two-body reaction is a reaction that has two reactants, i.e., the sum of the stoichiometric coefficients of the reactants equals exactly two. Reversible two-body reactions have stoichiometric coefficients that sum to exactly two on one side of the reaction and no more than two on the other. We now show how conservation constraints given by the stoichiometry of Eq. (17) allow the system of four ODEs describing the evolution of the species concentrations $y_{A}, y_{B}, y_{C}$, and $y_{D}$ to be replaced by a single ODE for a scalar progress variable $\lambda$. The natural progress variable for Eq. (17) obeys

$$
\frac{d \lambda}{d t}=k_{f} y_{A} y_{B}-k_{b} y_{C} y_{D}
$$

and changes in the concentrations satisfy

$$
\begin{aligned}
& \Delta y_{A}=\Delta y_{B}=-\lambda, \\
& \Delta y_{C}=\Delta y_{D}=\lambda .
\end{aligned}
$$


Writing each concentration in Eq. (25) as the sum of its initial value and its change, this ODE becomes

$$
\frac{d \lambda}{d t}=c \lambda^{2}+b \lambda+a
$$

for $a, b$, and $c$ given by

$$
\begin{aligned}
& a=k_{f} y_{A}^{0} y_{B}^{0}-k_{b} y_{C}^{0} y_{D}^{0} \\
& b=-k_{f}\left(y_{A}^{0}+y_{B}^{0}\right)-k_{b}\left(y_{C}^{0}+y_{D}^{0}\right) \\
& c=k_{f}-k_{b}
\end{aligned}
$$

Any two-body reaction leads to an ODE of the form of $\mathrm{Eq}(28)$ for some $a, b$, and $c$, even if the reaction does not match Eq. (17) exactly.

If $c=0$, the solution of Eq. (28) is an exponential given by

$$
\lambda(t)=\frac{a}{b}\left(1-e^{-b t}\right),
$$

and $-1 / b$ can be used as a time constant for the reaction. For nonzero $c$, the solution of Eq. (28) depends upon the sign of $q$, defined by

$$
q \equiv 4 a c-b^{2} .
$$

If the rate constants $k_{f}$ and $k_{b}$ are positive and the concentrations are nonnegative, then $q \leq 0$. The only case for which the equality $q=0$ holds is if all initial concentrations are zero. ${ }^{19}$ The solution of Eq. (28) for $q<0$ and nonzero $c$ is ${ }^{20}$

$$
\lambda(t)=-\frac{1}{2 c}\left(b+\sqrt{-q} \frac{1+\phi \exp (-\sqrt{-q} t)}{1-\phi \exp (-\sqrt{-q} t)}\right)
$$

in which $\phi$ is given by

$$
\phi=\frac{b+\sqrt{-q}}{b-\sqrt{-q}} .
$$

The parameter $\phi$ lies within the range $-1 \leq \phi<1$, so the denominator $1-\phi \exp (-\sqrt{-q} t)$ is strictly nonzero. The equilibrium $(t \rightarrow \infty)$ solution is identified as

$$
\lambda_{e q}=-\frac{1}{2 c}(b+\sqrt{-q}) .
$$

Note that if the initial conditions are in equilibrium, then $a=0, \sqrt{-q}=b$, and $\phi=0$.
A single timescale, $\tau_{r}$, defined by

$$
\tau_{r} \equiv 1 / \sqrt{-q},
$$

is identified for the reaction. Typically, a fast reaction is defined as one with timescale $\tau_{r}$ smaller than $\epsilon_{f} \Delta t$, where $\epsilon_{f}$ is a user-defined scale factor.

The proximity of the reaction to equilibrium can be determined by comparing the solution at $t=\Delta t$ to the equilibrium $(t \rightarrow \infty)$ solution. If the $t=\Delta t$ solution is within some specified tolerance of the equilibrium solution for all of the species, the reaction is considered close to equilibrium. That is, the reaction is close to equilibrium if

$$
\frac{\left\|y_{i}(\Delta t)-y_{i, e q}\right\|}{y_{i, e q}} \leq \epsilon_{e q} y_{i, e q}
$$

for $i$ corresponding to each of the species involved in the reaction and $y_{i, e q}$ denoting the equilibrium value of $y_{i}$. The parameter $\epsilon_{e q}$ is specified by the user and can take different values for the various species. This constraint can be written in terms of $\lambda$ provided the most restrictive species constraint is first identified and then written in terms of the progress variable. If $\epsilon_{e q}$ is the same for all species (as in the current work), then the most restrictive constraint in terms of $\lambda$ is generally the species that has the smallest equilibrium concentration.

\subsection{Three-body reactions}

For our purposes here, three-body reactions are identified by summing the stoichiometric coefficients on either side of the reaction equation. If one side sums to three and the other side sums to three or less, the reaction is three body. The following are examples of three-body reactions:

$$
\begin{aligned}
& 3 A \rightleftarrows B, \\
& A+B+C \rightleftarrows D+E, \\
& 2 A+B \rightleftarrows C+D, \\
& A+B+M \rightleftarrows C+D+M .
\end{aligned}
$$

Three-body reactions can be represented with a single ODE using the same conservation ideas employed in the previous section. However, this ODE has a source term which is cubic in the dependent 
variable, not quadratic as in the two-body case. Therefore, the ODE takes the form

$$
\frac{d x}{d t}=\delta x^{3}+\gamma x^{2}+\beta x+\alpha
$$

for some constants $\delta, \gamma, \beta$, and $\alpha$, and for $x$ being an appropriate choice of species concentration or progress variable.

The derivation of a timescale for Eq. (43) is found in Ref. ${ }^{19}$ In the current work, a timescale for Eq. (43) is approximated by using the the initial value $x_{0}$ to combine the cubic and quadratic terms into an effective quadratic term:

$$
\begin{aligned}
& \frac{d x}{d t}=d x^{3}+c x^{2}+b x+a \\
& =c^{\prime} x^{2}+b x+a .
\end{aligned}
$$

In Eq. (45),

$$
c^{\prime}=d x_{0}+c .
$$

The error in assuming that the cubic source term is quadratic decreases exponentially as equilibrium is approached.

The fact that the rate constants change in time has been neglected in the previous developments. Rate constants are a function of the thermodynamic state, which will change as the reaction progresses toward equilibrium. The timescale calculation is largely unaffected by this variation since it is an instantaneous indicator at the given conditions, although the solution given by Eq. (21) which leads to Eq. (37) for the timescale $\tau_{r}$ is not exact if $k_{f}$ and $k_{b}$ vary with time. However, the purpose of these calculations - to quantify the speed of the reactions and their proximity to equilibrium - can be accomplished despite these limitations. Each reaction is studied as if it were acting alone. When the equilibrium constraints are imposed, the interaction of the reactions must be taken into account, and the dependence of the rate constants on the thermodynamic state can also be included if desired.

\section{Linear Independence and Conservation Constraints}

Imposing equilibrium requires adjusting the species concentrations to satisfy the algebraic equilibrium constraints in a manner consistent with the conservation constraints. Ramshaw et al. ${ }^{6,7}$ developed an iteration scheme for finding the progress variables for the set of equilibrium reactions so that conservation was guaranteed. However, as Rein indicates, ${ }^{10}$ Ramshaw did not eliminate redundant equilibrium constraints from his system, ${ }^{5}$ and this can lead to poor convergence of the equilibrium system.

Rein took the additional step of designating independent and dependent species based on global conservation constraints. ${ }^{10}$ Rein's method then imposes equilibrium in two steps. First, it finds the concentrations of the dependent species using the equilibrium constraints and a set of conserved scalars for the equilibrium reactions projected onto the dependent variables. It then finds the concentrations of the independent species using the global conservation constraints. This approach has a drawback, however: if an independent species appears in an equilibrium reaction, then these two solution steps are not independent.

Consider, for example, the reactions

$$
\begin{aligned}
& \mathrm{H}_{2}+\mathrm{O} \rightleftarrows \mathrm{OH}+\mathrm{H} \\
& \mathrm{H}_{2}+\mathrm{OH} \rightleftarrows \mathrm{H}_{2} \mathrm{O}+\mathrm{H} \\
& 2 \mathrm{OH} \rightleftarrows \mathrm{H}_{2} \mathrm{O}+\mathrm{O}
\end{aligned}
$$

which are important for hydrogen combustion and common to hydrocarbon combustion problems. If all three reactions are in equilibrium, the constraints they impose are (using brackets to denote concentrations)

$$
\begin{aligned}
& \frac{[\mathrm{OH}][\mathrm{H}]}{\left[\mathrm{H}_{2}\right][\mathrm{O}]}=K_{1} \\
& \frac{\left[\mathrm{H}_{2} \mathrm{O}\right][\mathrm{H}]}{\left[\mathrm{H}_{2}\right][\mathrm{OH}]}=K_{2} \\
& \frac{\left[\mathrm{H}_{2} \mathrm{O}\right][\mathrm{O}]}{[\mathrm{OH}]^{2}}=K_{3}
\end{aligned}
$$

for equilibrium constants $K_{1}, K_{2}$, and $K_{3}$. These three reversible reactions involve five species, $\left\{\mathrm{H}, \mathrm{O}, \mathrm{H}_{2}, \mathrm{OH}, \mathrm{H}_{2} \mathrm{O}\right\}$, which are composed of two elements, $H$ and $O$. Including conservation constraints for $H$ and $O$, there are five equations for the five unknown species concentrations. However, Eq. (52) can be written in terms of the other two 
equilibrium constraints,

$$
K_{3}=\frac{\left[H_{2} O\right][O]}{[O H]^{2}}=\frac{\frac{\left[H_{2} O\right][H]}{\left[H_{2}\right][O H]}}{\frac{[O H][H]}{\left[H_{2}\right][O]}}=\frac{K_{2}}{K_{1}}
$$

so the constraints are not independent. Since the equilibrium constraints are redundant, the fiveequation system constructed in such a manner would be indeterminate. A method such as Ramshaw's has poor convergence characteristics if the redundant constraint is not removed, and some methods would not converge at all.

Additionally, since all of the species are involved in the equilibrium, no segregation of the species into dependent and independent groups will lead to two independent calculations by Rein's method. For example, if one picks $H$ and $O$ as the independent species, Rein's method first constructs a conserved scalar for the equilibrium modes involving $\mathrm{H}_{2}, \mathrm{OH}$, and $\mathrm{H}_{2} \mathrm{O}$, and then solves a system of three equations for $\left\{\mathrm{H}_{2}, \mathrm{OH}, \mathrm{H}_{2} \mathrm{O}\right\}$ using two of the equilibrium constraints and this conserved scalar. The global conservation constraints are then used to calculate the concentrations of $H$ and $O$ after the other three concentrations are known. However, the concentrations of $H$ and $O$ appear in the equilibrium constraints, so the system for $\left\{\mathrm{H}_{2}, \mathrm{OH}, \mathrm{H}_{2} \mathrm{O}\right\}$ is not closed.

The key is that these reactions provide an additional conservation constraint,

$$
\left[H_{2}\right]+[H]=\text { constant }
$$

that follows from the stoichiometry of the three reactions but does not match a classic conservation constraint such as element conservation. This constraint is easily found by constructing the orthogonal complement to the reaction subspace, as described in Section 2. The algebraic system that should be solved for the five species concentrations takes two constraints from Eqs. (50) through (52), two constraints from conservation of $H$ and $O$, and the additional constraint in Eq. (54). An alternative to this five-equation system is to write equilibrium constraints for the progress variables of two of the reactions, which reduces the problem to two coupled equations. This approach is described in Section 6 .

\section{Imposing the Equilibrium Constraints}

In addition to the methods described above for identifying linearly independent equilibrium reactions, we introduce a new equilibrium constraint in Subsection 6.2. The traditional constraint is presented first for comparison.

\subsection{Imposing the Constraint $F=0$}

Consider a set of $k^{\prime}$ linearly independent equilibrium reactions involving $n$ species $y_{i}$, each with reaction vector $\mathbf{r}^{i}$, source term $F_{i}$, and progress variable $\lambda_{i}$. The concentrations and source terms can be placed into vectors $\mathbf{y}$ and $\mathbf{F}$ in which component $i$ corresponds to species $i$. The progress variable is governed by

$$
\frac{d \lambda_{i}}{d t}=F_{i} \quad 1 \leq i \leq k^{\prime}
$$

Imposing the traditional equilibrium constraint $F_{i}=$ 0 requires finding $\left\{\lambda_{i}\right\}, 1 \leq i \leq k^{\prime}$, such that

$$
\mathbf{F}\left(\mathbf{y}_{e q}\right)=(0) \quad \text { for } \quad \mathbf{y}_{e q}=\mathbf{y}^{0}+\sum_{j=1}^{k^{\prime}} \lambda_{j} \mathbf{r}^{j}
$$

given initial concentrations $\mathbf{y}^{0}$.

As discussed in, ${ }^{1}$ applying Newton's method to Eq. (56) requires solving

$$
\left(\frac{\partial \mathbf{F}}{\partial \boldsymbol{\lambda}}\right)\left(\Delta \boldsymbol{\lambda}^{l}\right)=\left(-\mathbf{F}^{l}\right) \text {. }
$$

The superscript $l$ indicates values at the $l^{t h}$ iteration, and the iteration is stopped after the values $\left\{F_{i}^{l}\right\}$ or the changes in the progress variables $\left\{\Delta \lambda_{i}^{l}\right\}$ fall below specified tolerances. The components of the derivative matrix $(\partial \mathbf{F} / \partial \boldsymbol{\lambda})$ are given by

$$
\left(\frac{\partial \mathbf{F}}{\partial \boldsymbol{\lambda}}\right)_{i, j}=\frac{\partial F_{i}}{\partial \lambda_{j}}
$$

Equation (56) is solved iteratively by calculating the $(l)^{t h}$ iterates $\left\{\Delta \lambda_{i}^{l}\right\}$ from Eq. (57), then using

$$
\lambda_{i}^{l+1}=\lambda_{i}^{l}+\Delta \lambda_{i}^{l} \quad 1 \leq i \leq n
$$


to calculate $\mathbf{F}\left(\mathbf{y}^{l+1}\right)$ for the next iteration for

$$
\mathbf{y}^{l+1}=\mathbf{y}^{0}+\sum_{j=1}^{k} \lambda_{j}^{l+1} \mathbf{r}^{j} .
$$

The elements of the derivative matrix given in Eq. (58) are constructed from the partial derivatives of the source terms with respect to the species concentrations. This construction uses the reaction vectors and is presented in detail by Mott. ${ }^{19}$

Although the elements of the matrix Eq. (58) vary with $\lambda_{i}$, in general they need only be calculated once per timestep. The iteration will still converge quickly, and using the initial values of these entries will save the significant expense of recalculating them.

\subsection{New Equilibrium Constraint}

Equation (57) strictly imposes $F_{i}=0$ for each of the source terms. The source term, however, is not identically zero as the system evolves. The condition $F_{i}=0$ is an approximation indicating that the difference between the production and loss terms is very small compared to the magnitude of these two terms. The value of $\lambda_{i}$ provides a measure of how hard the equilibrium reaction $i$ must work in order to counteract the changes produced by the other reactions that disturb its equilibrium. Since $\lambda_{i}=0$ initially, a discreet form of Eq. (55) gives

$$
\frac{\lambda_{i}}{\Delta t}=\overline{F_{i}}
$$

for the average value, $\overline{F_{i}}$, that the source term must take in order to produce the given value of $\lambda_{i}$.

Therefore the new equilibrium constraint uses $\overline{F_{i}}$ as the target value of the source term rather than zero, and the system that must be solved is

$$
\left(\frac{\partial \mathbf{F}}{\partial \boldsymbol{\lambda}}\right)\left(\Delta \boldsymbol{\lambda}^{l}\right)=\left(\overline{\mathbf{F}}^{l}-\mathbf{F}^{l}\right) .
$$

To make the iteration more implicit, the iterate $\bar{F}_{i}^{l}$ is given by

$$
{\overrightarrow{F_{i}}}^{l}=\frac{\lambda_{i}^{l}+\Delta \lambda_{i}^{l}}{\Delta t}
$$

so that rearranging the system gives

$$
\left(\frac{\partial \mathbf{F}}{\partial \boldsymbol{\lambda}}-\frac{1}{\Delta t} I\right)\left(\Delta \boldsymbol{\lambda}^{l}\right)=\left(\frac{1}{\Delta t} \lambda^{l}-\mathbf{F}^{l}\right) .
$$

for identity matrix $I$. This system also shows very good convergence characteristics, needing at most two or three iterations in the testing done to date. Using Eq. (64) allows an equation to be replaced with an algebraic equilibrium constraint farther from equilibrium than with the traditional $F_{i}=0$ constraint.

A search of the partial-equilibrium literature identified no published methods that identify a set of independent equilibrium constraints and impose these constraints directly on the system. The method of Ramshaw et al., ${ }^{6,7}$ for imposing equilibrium through the progress variables could be used instead of the Newton iteration shown here. Redundant constraints should be identified and removed before any method is used to impose the equilibrium. To our knowledge, imposing an average value for $F$ rather than $F=0$ is original to the current work.

\section{Integration Results: Ther- monuclear Mechanism}

The new PE tools are applied to an extremely stiff thermonuclear mechanism which the stiff integrator CHEMEQ2 ${ }^{19,22}$ cannot solve. CHEMEQ2 (name derived from CHEMical EQuation solver, not equilibrium) is designed to integrate stiff ODE systems within a process-split reacting-flow code. As such, CHEMEQ2 is a second-order method with low overhead and start-up costs that works well for problems typical of hydrocarbon combustion.

A set of 13 nuclear species $\left({ }^{4} \mathrm{He},{ }^{12} \mathrm{C},{ }^{16} \mathrm{O},{ }^{20} \mathrm{Ne}\right.$, ${ }^{24} \mathrm{Mg},{ }^{28} \mathrm{Si},{ }^{32} \mathrm{~S},{ }^{36} \mathrm{Ar},{ }^{40} \mathrm{Ca},{ }^{44} \mathrm{Ti},{ }^{48} \mathrm{Cr},{ }^{52} \mathrm{Fe}$, and ${ }^{56} \mathrm{Ni}$ ) interact through the 18 reversible reactions listed in Table 7. This system is used in hydrodynamic simulations of supernovae explosions which exhibit flame- and detonation-like behavior. The medium is a dense degenerate plasma which acts thermodynamically close to a polytropic gas with $\gamma=1.4$. The details of the reaction mechanism and the equation of state are discussed by Khokhlov. ${ }^{21}$ The current test problems begin with $50 \%$ oxygen and $50 \%$ carbon by moles at initial temperature 
Table 7: Thermonuclear Reaction Mechanism.

\begin{tabular}{cccccc}
\hline \hline 1$)$ & & & $3{ }^{4} \mathrm{He}$ & $\rightleftarrows$ & ${ }^{12} \mathrm{C}$ \\
$2)$ & ${ }^{12} \mathrm{C}$ & + & ${ }^{12} \mathrm{C}$ & $\rightleftarrows$ & ${ }^{4} \mathrm{He}$ \\
& & & & + & ${ }^{20} \mathrm{Ne}$ \\
$3)$ & ${ }^{12} \mathrm{C}$ & + & ${ }^{12} \mathrm{C}$ & $\rightleftarrows$ & ${ }^{24} \mathrm{Mg}$ \\
$4)$ & ${ }^{12} \mathrm{C}$ & + & ${ }^{16} \mathrm{O}$ & $\rightleftarrows$ & ${ }^{4} \mathrm{He}$ \\
& & & & + & ${ }^{24} \mathrm{Mg}$ \\
$5)$ & ${ }^{12} \mathrm{C}$ & + & ${ }^{16} \mathrm{O}$ & $\rightleftarrows$ & ${ }^{28} \mathrm{Si}$ \\
$6)$ & ${ }^{16} \mathrm{O}$ & + & ${ }^{16} \mathrm{O}$ & $\rightleftarrows$ & ${ }^{4} \mathrm{He}$ \\
& & & & + & ${ }^{28} \mathrm{Si}$ \\
$7)$ & ${ }^{16} \mathrm{O}$ & + & ${ }^{16} \mathrm{O}$ & $\rightleftarrows$ & ${ }^{32} \mathrm{~S}$ \\
$8)$ & ${ }^{4} \mathrm{He}$ & + & ${ }^{12} \mathrm{C}$ & $\rightleftarrows$ & ${ }^{16} \mathrm{O}$ \\
$9)$ & ${ }^{4} \mathrm{He}$ & + & ${ }^{16} \mathrm{O}$ & $\rightleftarrows$ & ${ }^{20} \mathrm{Ne}$ \\
$10)$ & ${ }^{4} \mathrm{He}$ & + & ${ }^{20} \mathrm{Ne}$ & $\rightleftarrows$ & ${ }^{24} \mathrm{Mg}$ \\
$11)$ & ${ }^{4} \mathrm{He}$ & + & ${ }^{24} \mathrm{Mg}$ & $\rightleftarrows$ & ${ }^{28} \mathrm{Si}$ \\
$12)$ & ${ }^{4} \mathrm{He}$ & + & ${ }^{28} \mathrm{Si}$ & $\rightleftarrows$ & ${ }^{32} \mathrm{~S}$ \\
$13)$ & ${ }^{4} \mathrm{He}$ & + & ${ }^{32} \mathrm{~S}$ & $\rightleftarrows$ & ${ }^{36} \mathrm{Ar}$ \\
$14)$ & ${ }^{4} \mathrm{He}$ & + & ${ }^{36} \mathrm{Ar}$ & $\rightleftarrows$ & ${ }^{40} \mathrm{Ca}$ \\
$15)$ & ${ }^{4} \mathrm{He}$ & + & ${ }^{40} \mathrm{Ca}$ & $\rightleftarrows$ & ${ }^{44} \mathrm{Ti}$ \\
$16)$ & ${ }^{4} \mathrm{He}$ & + & ${ }^{44} \mathrm{Ti}$ & $\rightleftarrows$ & ${ }^{48} \mathrm{Cr}$ \\
$17)$ & ${ }^{4} \mathrm{He}$ & + & ${ }^{48} \mathrm{Cr}$ & $\rightleftarrows$ & ${ }^{52} \mathrm{Fe}$ \\
$18)$ & ${ }^{4} \mathrm{He}$ & + & ${ }^{52} \mathrm{Fe}$ & $\rightleftarrows$ & ${ }^{56} \mathrm{Ni}$ \\
\hline \hline
\end{tabular}

$5 \times 10^{9} \mathrm{~K}$ and density $1 \times 10^{7} \mathrm{gm} / \mathrm{cm}^{3}$. The integration is performed from time 0 to $10^{2}$ seconds. The effects of the partial equilibrium tools on the integration is demonstrated on a constant-temperature problem. The stiffness ratio (i.e., the ratio of the nonzero eigenvalues of the Jacobian matrix $\partial \mathbf{g} / \partial \mathbf{y}$ with the largest and smallest magnitudes) for this system increases from $\sim 10^{4}$ early in the simulation to over $10^{9}$ before equilibrium is reached.

The mechanism includes only one three-body reaction, and the timescale and proximity to equilibrium for this reaction is estimated using the quadratic approximation of Eq. (45). Equilibrium is imposed in some cases based solely on the reaction timescale $\tau_{r}$, while in others both $\tau_{r}$ and the proximity of the reaction to equilibrium are checked. The $\lambda_{e q}$ constraint refers to this proximity check using Eqs. (36) and (38). The timestep is used in Eq. (38) with $\epsilon_{e q}=0.01$ for all species, so in the current simulations a reaction is "close" to equilibrium when each of its species will be within one percent of their equilibrium values by the end of the timestep. The equilibrium reactions are then removed from the ki-

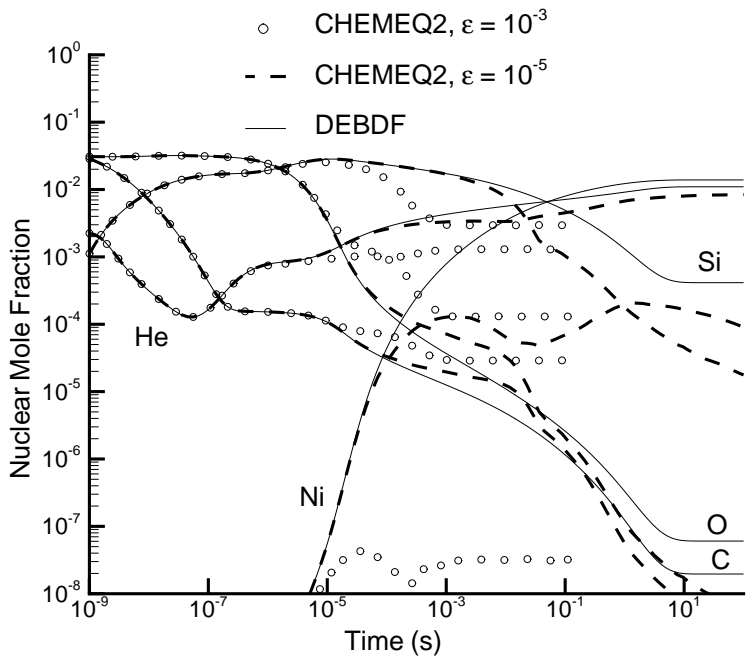

Figure 1: Nuclear mole fraction histories obtained using DEBDF and using CHEMEQ2 with no PE imposed. The user-specified parameter $\varepsilon$ dictates the timestep by monitoring the relative error in the integration.

netic integration, and the constraints imposed in a second step.

The non-equilibrium reactions are integrated using the $\alpha$-QSS method as implemented in CHEMEQ2. ${ }^{19,22,23}$ This method works well for systems typical in hydrocarbon combustion, but it is not well suited for extremely stiff problems. A reference solution is calculated using the subroutine DEBDF, which employs a variable-order Gear method as implemented in LSODE. DEBDF is part of SLATEC, a library of computational subroutines available on Silicon Graphics and Cray computers. ${ }^{24}$ Since our goal is implementation in a reacting-flow code, the performance of the PE tools in conjunction with CHEMEQ2 is desired, but a standard Gear method performs very well on this problem when not coupled to a flow problem. Figure 1 illustrates the reference solution obtained using DEBF and two inaccurate solutions obtained using CHEMEQ2 alone. All mole fractions are presented as "nuclear mole fractions," given by

species $i$ nuclear mole fraction $=$ species $i$ mole fraction $\overline{\text { mass number of the nucleus of species } i}$. 

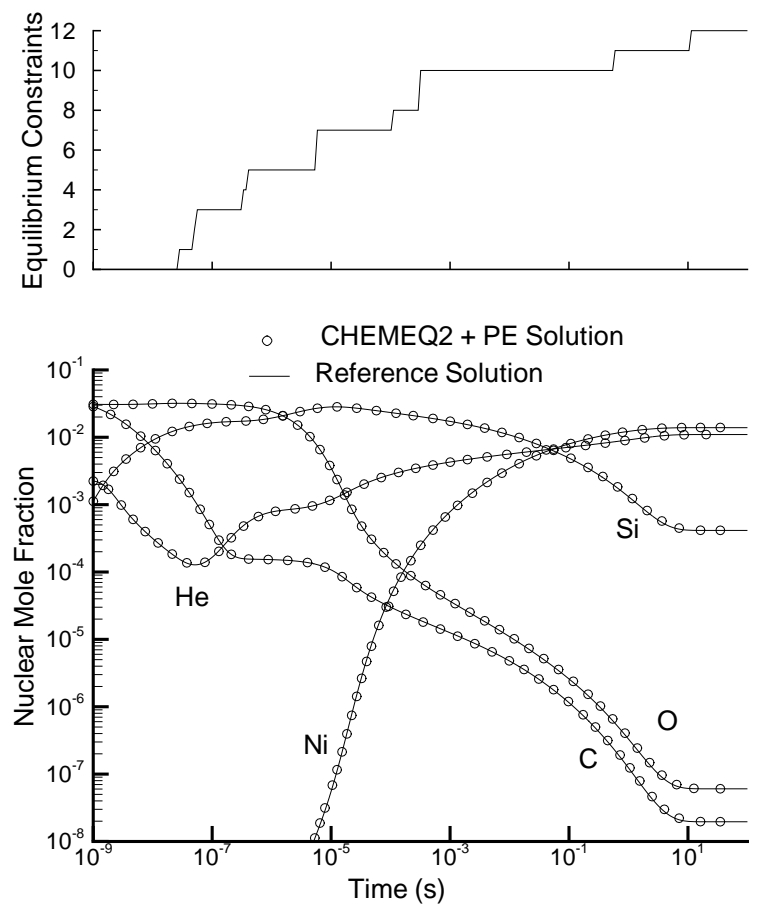

Figure 2: Nuclear mole fraction histories and the number of independent equilibrium reactions for the constant-temperature thermonuclear test problem.

For instance, the initial mole fractions are 0.5 for both ${ }^{12} \mathrm{C}$ and ${ }^{16} \mathrm{O}$, so the initial nuclear mole fractions for carbon and oxygen are $1 / 24$ and 1/32, respectively.

Figures 2 and 3 show results obtained using CHEMEQ2 and the new PE methods.. Nuclear mole fractions for the reference solution are shown as solid lines, and the same variables obtained from the combined CHEMEQ2+PE code are included as open circles. For this case both $\tau_{r}$ and $\lambda_{e q}$ switches are used for determining equilibrium, with $\epsilon_{f}=1$ (i.e., a reaction is "fast" if $\tau_{r}<\Delta t$ ). The CHEMEQ2+PE results show very good agreement with the reference solution..

Figures 2 and 3 also show the number of independent equilibrium constraints imposed by the algorithm as a function of time. These curves are identical in the two plots and are included in both for easy reference to the mole fraction plots. The nu-
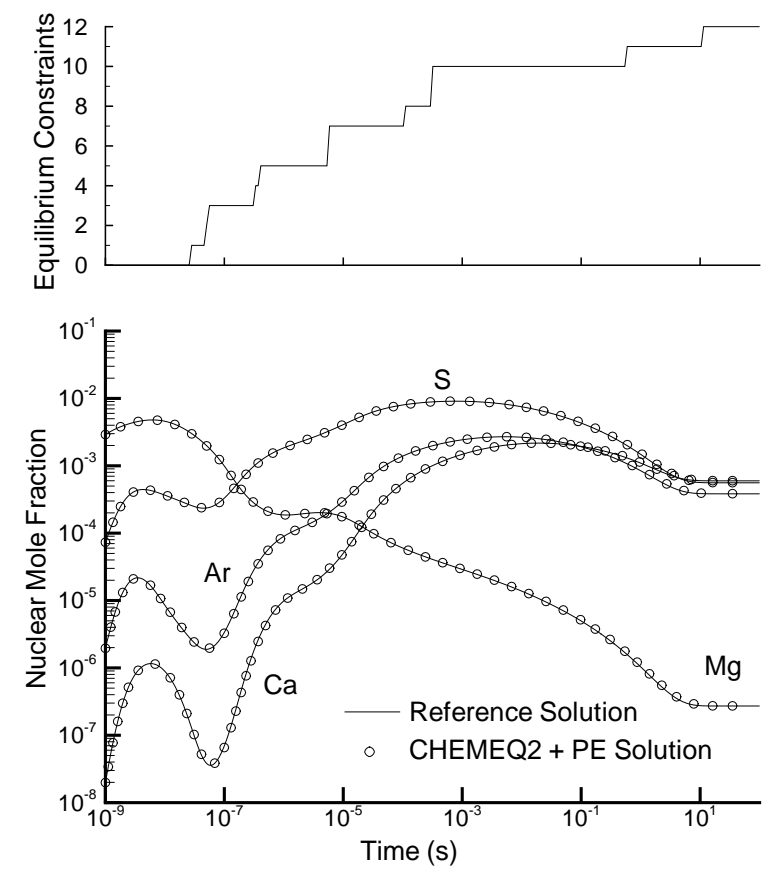

Figure 3: Additional nuclear mole fraction histories and the number of independent equilibrium reactions for the constant-temperature thermonuclear test problem.

clear mechanism is unusual in that only one global conservation law holds for the 13-species system. (This conservation law is for $\alpha$ particles; dividing the mass number of the species by 4 gives the number of $\alpha$-particles per particle of that species.) A typical hydrocarbon combustion problem with $n$ species composed of $n_{e}$ elements will have $n_{e}$ global conservation constraints from element conservation and a maximum of $n-n_{e}$ independent equilibrium constraints. Therefore, the nonlinear system that imposes equilibrium will never involve more than $n-n_{e}$ equations. Since only one conservation law holds for the nuclear network, a maximum of 12 independent equilibrium constraints is possible. As Figs. 2 and 3 show, no equilibrium is present initially, and this maximum value of 12 is reached when the system as a whole reaches equilibrium.

To study the effectiveness of the new tools, a se- 

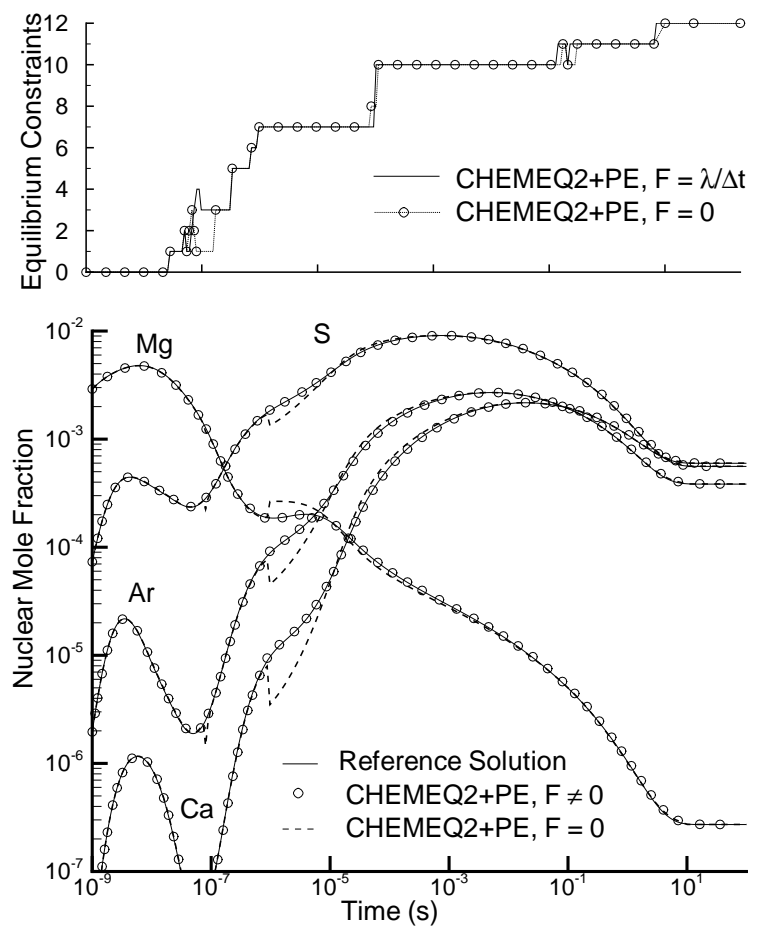

Figure 4: Thermonuclear results comparing the use of the constraint $F=0$ to the constraint $F=\lambda / \Delta t$, for $\epsilon_{f}=1$ and the $\tau_{r}$-based switch only.

ries of tests are presented using variations on the base test presented above. The first variation is presented in Fig. 4, in which solutions are shown using only the $\tau_{r}$-based criteria for equilibrium and both the constraint $F=0$ and the constraint $F=\lambda / \Delta t$. In both sets of calculations $\epsilon_{f}=1$. The results obtained using the constraint $F=\lambda / \Delta t$ are very similar to the results in Fig. 3 using both the $\tau_{r}$ and $\lambda_{e q}$ criteria for identifying equilibrium. The number of equilibrium modes found using the $\tau_{r}$-based requirement alone is not as stable, showing nonmonotone behavior around $10^{-7}$ and $10^{-1} \mathrm{~s}$. These oscillations are not seen when the $\lambda_{e q}$ requirement is used in combination with the $\tau_{r}$-based requirement. The species solution, however, is not adversely affected by these oscillations.

A more dramatic difference is seen in the mole fraction curves when the constraint $F=0$ is used instead of the new constraint. Jumps are seen in the mole fraction curves when the system adds the seventh equilibrium constraint at approximately $10^{-6} \mathrm{~s}$. A similar but less substantial discontinuity is seen where the $F=0$ calculation adds its third equilibrium constraint. The seventh equilibrium constraint is more illustrative because both the $F=0$ and $F=\lambda / \Delta t$ calculations add this constraint at the same time. The first six equilibrium reactions are Reactions $10-15$ as numbered in Table 7 , and the seventh equilibrium reaction is Reaction 9:

$$
\mathrm{He}+\mathrm{O} \rightleftarrows \mathrm{Ne} \text {. }
$$

The species shown in Fig. 4 are not directly involved in Reaction 9 , but they are involved in the other six reactions for which equilibrium is imposed at this point. Reaction 9 is coupled to the other equilibrium reactions through $\mathrm{Ne}$, which also appears in Reaction 10. Adding Reaction 9 to the equilibrium system at this point using the constraint $F=0$ is premature and causes substantial errors in the solution. Although Reaction 9 is fast as measured by the $\tau_{r}$ criterion, substantial changes in the species concentrations are required in order to satisfy $F=0$ for it and the other equilibrium reactions. Imposing equilibrium as soon as the timescale is smaller than the timestep is therefore premature when using the constraint $F=0$; Using the $F=\lambda / \Delta t$ constraint smoothes this transition and maintains an accurate solution.

This effect is more dramatic as the value of $\epsilon_{f}$ is increased. Results obtained using $\epsilon_{f}=10$ are included in Fig. 5. Using $\epsilon_{f}=10$ with the $\tau_{r}$-based switch alone is not particularly reasonable; one would not assume that imposing an equilibrium constraint as soon as $\tau_{r}<10 \Delta t$ would provide an accurate result. This prediction is validated by the results obtained using the $F=0$ constraint. As equilibrium is inappropriately applied early in the integration, wild oscillations occur in the species mole fractions. The equilibrium calculation causes changes in the species concentration so great that the subsequent timescale calculation produces a $\tau_{r}$ value that no longer satisfies the equilibrium criterion. Additionally, a jump in the mole fractions is seen at $t \approx 1$ as the final equilibrium values are imposed prematurely.

Applying the $F=\lambda / \Delta t$ constraint, even with the unreasonable $\epsilon_{f}=10$ criterion, results in an acceptable solution. The mole fraction curve for argon is 

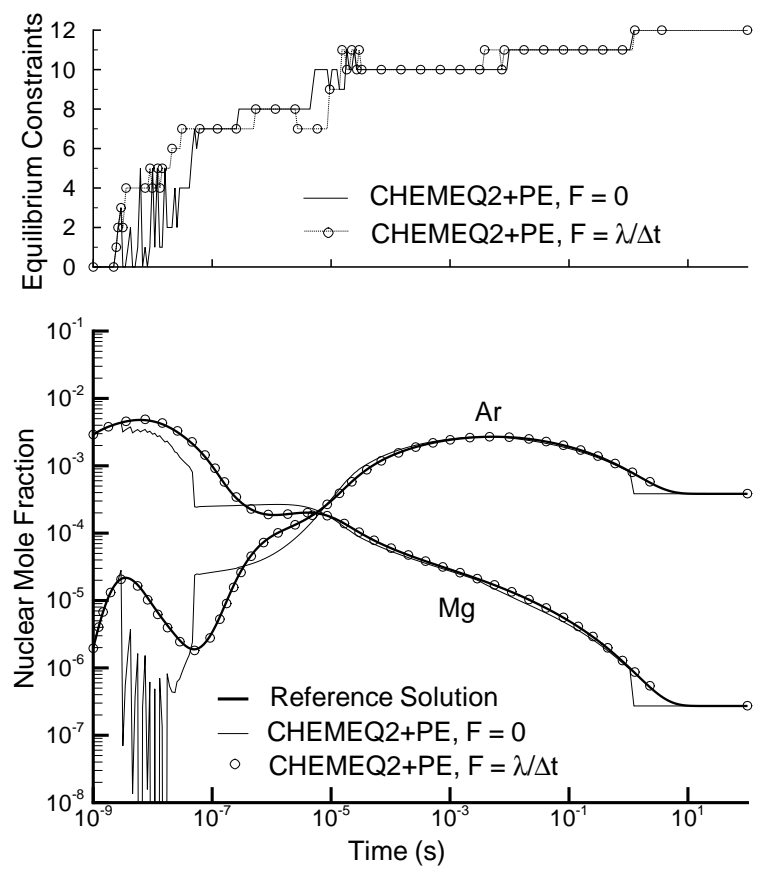

Figure 5: Thermonuclear results comparing the use of the constraint $F=0$ to the constraint $F=\lambda / \Delta t$, for $\epsilon_{f}=10$ and the $\tau_{r}$-based switch only.

seen to deviate from the reference solution early in the integration, but overall agreement with the reference solution is very good. The $F=\lambda / \Delta t$ constraint makes the equilibrium imposition more robust and allows for accurate solutions to be calculated for a wider range of the equilibrium criterion. This property is important in reacting flow applications since conditions will vary greatly throughout the flowfield. If the parameters required to get a suitable solution are too restrictive, only parts of the flowfield or the integration could be calculated properly.

Since the errant results in Fig. 5 resulted from a premature application of equilibrium, perhaps including the $\lambda_{e q}$ criterion in identifying equilibrium can also eliminate the problem. Returning to the traditional $F=0$ constraint, Fig. 6 compares results obtained using the $\tau_{r}$ criterion alone to results obtained when the $\lambda_{e q}$ test is also included. The results obtained using the $\tau_{r}$-based switch for
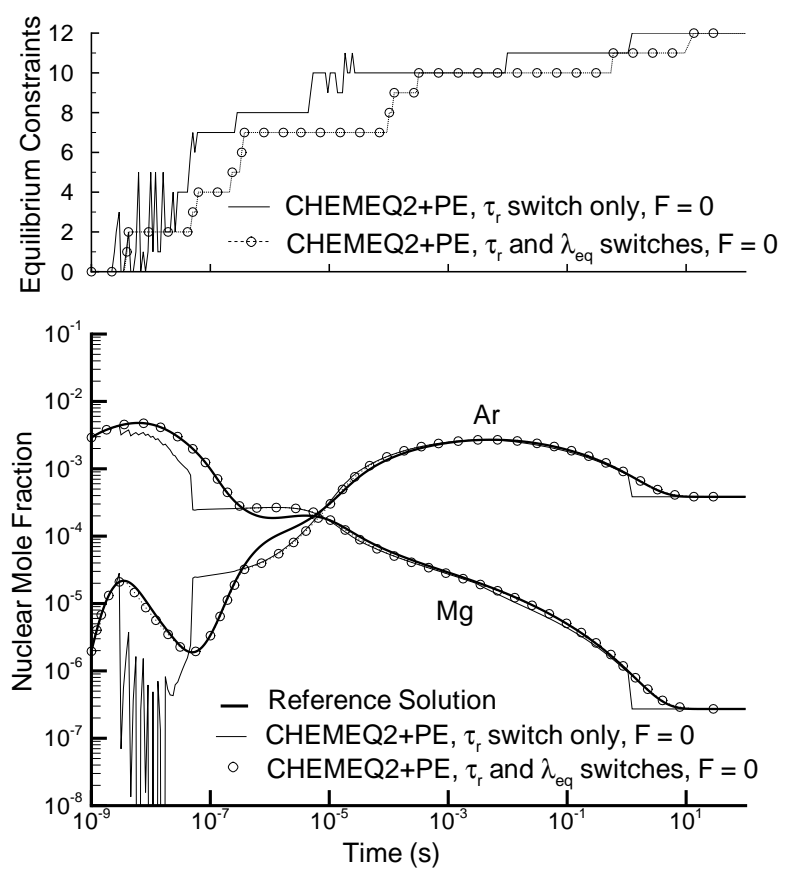

Figure 6: Thermonuclear results for the constraint $F=0$ comparing the use of both the $\lambda_{e q}$ switch and the $\tau_{r}$-based switch to the use of the $\tau_{r}$-based switch alone for $\epsilon_{f}=10$.

$\epsilon_{f}=10$ and $F=0$ first seen in Fig. 5 are repeated in Fig. 6. Including the $\lambda_{e q}$ switch with $\epsilon_{e q}=0.01$ eliminates the oscillations early in the integration by recognizing that imposing equilibrium (especially of the form $F=0$ ) is not yet appropriate. The number of equilibrium modes found by the code is also much more stable: changes in the mole fractions resulting from imposing equilibrium will not push the timescale back above the equilibrium threshold.

Note, however, that after $10^{-7} \mathrm{~s}$ the solution including the $\lambda_{e q}$ switch deviates from the reference solution and follows the solution obtained using the $\tau_{r}$ switch alone. The solution including the $\lambda_{e q}$ switch returns to the reference solution by $10^{-3} \mathrm{~s}$ and does not impose the final equilibrium prematurely. As in Fig. 4, the problematic constraint after $10^{-7} \mathrm{~s}$ is the seventh one imposed, again the one for Reaction 9 given in Eq. (66). The $\tau_{s}$ criterion for this reaction 

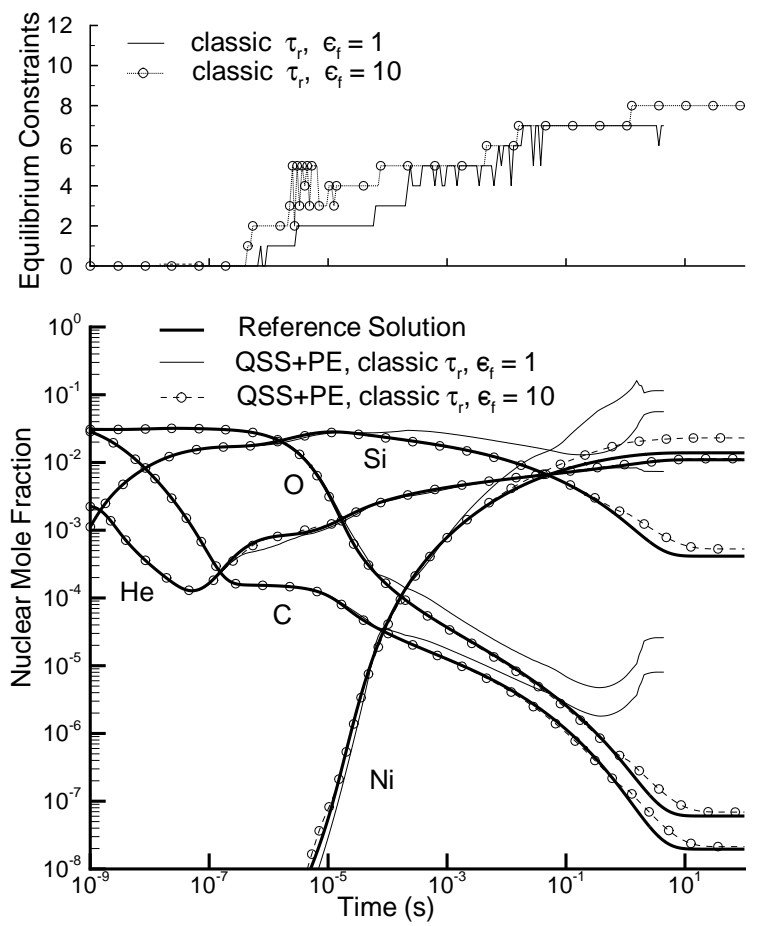

Figure 7: Thermonuclear results using the Section 3 timescale calculation for $\epsilon_{f}=1$ and $\epsilon_{f}=10$ using the $\tau_{r}$-based switch only and the constraint $F=0$.

is more restrictive, so adding the $\lambda_{e q}$ switch does not alter the point at which it is classified as being in equilibrium. Using a more restrictive $\lambda_{e q}$ constraint could delay imposing this equilibrium, but recall that we already have a solution for this problem: using $F=\lambda / \Delta t$ produced an accurate solution even for $\epsilon_{f}=10$. We also know that if we wait too long to impose equilibrium the kinetic integrator will begin to introduce errors into the solution. Therefore, the $\lambda_{e q}$ switch cannot serve as a replacement for using the constraint $F=\lambda / \Delta t$ in extending the range over which the equilibrium can be imposed.

How does the new reaction timescale calculation compare to the traditional method described in Section 3? The traditional method writes an ODE for each species in the reaction and takes the largest timescale from the group of ODEs to characterize the speed of the reaction. Results obtained using this method are included in Fig 7. These results are obtained using the $\tau_{r}$-based switch alone and the constraint $F=0$. Mole fractions obtained using $\epsilon_{f}=1$ are given by the thin, solid lines in Fig. 7 . These results deviate greatly from the reference solution at later times and experience the same premature freezing problem experienced by CHEMEQ2 acting alone. The number of equilibrium constraints found using $\epsilon_{f}=1$, given by the solid line in the top graph in Fig. 7, is much lower than in the previous solutions obtained using the new timescale calculations. The curves stop abruptly before $10 \mathrm{~s}$ because the calculation becomes prohibitively expensive. The equilibrium tools do not eliminate enough stiffness from the system, and the $\alpha$-QSS method is left with a system it still cannot integrate. Switching to the constraint $F=\lambda / \Delta t$ does not help in this case because the problem results from waiting too long to impose equilibrium, not from imposing equilibrium too early. In some problems this inability to recognize viable equilibrium would be expensive but not catastrophic, but in this case the kinetic integrator cannot integrate the system unless the PE method removes more of the stiffness from the system.

Increasing $\epsilon_{f}$ to 10 helps, as is seen in the curves marked with open circles in Fig. 7. The results are still not satisfactory, though. Minor deviations from the reference solution are seen early in the integration (such as in $\mathrm{Ni}$ around $10^{-5} \mathrm{~s}$ ). Larger errors are seen later in the integration. Again, these errors do not arise from an inaccurate application of equilibrium, so using the constraint $F=\lambda / \Delta t$ does not help. The errors arise because not enough equilibrium is recognized and imposed, leaving the kinetic integrator with a problem it cannot accurately solve. Even with $\epsilon_{f}=10$ the code believes that only eight modes are in equilibrium at the end of the integration. A comparison of the equilibrium mode histories using the traditional $\tau_{r}$ calculation and the new $\tau_{r}$ calculation is included in Fig. 8.

\section{Discussion and Summary}

The new techniques for identifying equilibrium and imposing a set of equilibrium constraints are more robust and accurate than traditional PE methods. They are better suited for a reacting flow calculation that can experience vastly different properties 


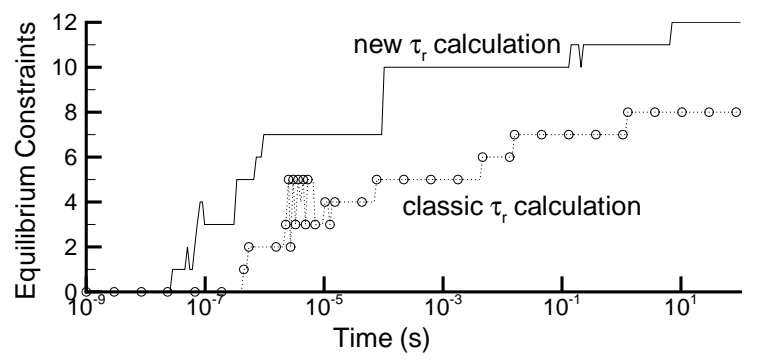

Figure 8: Number of independent equilibrium constraints for the thermonuclear problem obtained using the classic timescale calculation and $\epsilon_{f}=10$ versus the new $\tau_{r}$ calculation and $\epsilon_{f}=1$. $\tau_{r}$-based switch only.

at different points in the calculation. The new constraint, $F=\lambda / \Delta t$, allows equilibrium to be assumed earlier in the calculation while maintaining the accuracy of the solution. A thorough error analysis should be done to determine specifically how this new constraint affects the accuracy of the integration.

Woven into the PE tools is a method for identifying conserved scalars for an arbitrary reaction set. This method is based on the stoichiometry of the reactions and is independent of equilibrium assumptions. This method leads to a straightforward test for determining which reactions in a set will produce redundant equilibrium constraints, so that these constraints can be eliminated when equilibrium is imposed.

\section{Acknowledgements}

This work was funded by the Office of Naval Research. The authors would also like to thank Vadim Gamezo and Alexei Khokhlov for providing the thermonuclear mechanism, a framework for testing the new methods, and their expertise.

\section{References}

[1] J.D. Hoffman, Numerical Methods for Engineers and Scientists. McGraw-Hill, Inc., New York, 1992.

[2] J.D. Lambert, Numerical Methods for Ordinary Differential Systems; The Initial Value Problem. John Wiley \& Sons, Chichester, England, 1991.

[3] E.S. Oran, J.P. Boris, Numerical Simulation of Reactive Flow, second Edition. Elsevier Science Publishing Co., Inc., New York, 2001.

[4] F. A. Williams, Combustion Theory, The Fundamental Theory of Chemically Reacting Systems, 2nd edition, Benjamin/Cummings Publishing Company, Menlo Park, California, 1985.

[5] J. D. Ramshaw. "Partial chemical equilibrium in fluid dynamics", Physics of Fluids, 23(4), pp. 675-680, April 1980.

[6] J. D. Ramshaw, L. D. Cloutman. "Numerical Method for Partial Equilibrium Flow," Journal of Computational Physics 39, 1981, pp. 405-417.

[7] J. D. Ramshaw, A. A. Amsden. "Inclusion of Temperature Variations in a Numerical scheme for Partial Equilibrium Flow," Journal of Computational Physics 71, 1987, pp. 224-227.

[8] J. D. Ramshaw, C. H. Chang. "Iteration Scheme for Implicit Calculations of Kinetic and Equilibrium Chemical Reactions in Fluid Dynamics," Journal of Computational Physics 116, 1995, pp. 359-364.

[9] A. A. Amsden, J.D. Ramshaw, P. J. O'Rourke, and J.K. Dukowicz. "KIVA: A computer program for two- and three-dimensional fluid flows with chemical reactions and fuel sprays," Los Alamos National Laboratory Report No. LA10245-MS, 1985.

[10] M. Rein. "The partial-equilibrium approximation in reacting flows," Physics of Fluids A, 4 (5), 1992, pp.873 - 886 .

[11] S. H. Lam and D. A. Goussis. "The CSP Method for Simplifying Kinetics," International 
Journal on Chemical Kinetics, 26, pp. 461-486, 1994.

[12] S. H. Lam. "Reduced Chemistry Modelling and Sensitivity Analysis," lecture notes for Aerothermochemistry for Hypersonic Technology, 1994-1995 Lecture Series Programme, the Von Karman Institute For Fluid Dynamics, April 24-28, 1995.

[13] M. Valorani and D. A. Goussis. "Explicit TimeScale Splitting Algorithms for Stiff ODE's: Auto-Ignition Behind a Steady Shock," submitted for publication, Journal of Computational Physics, 1996.

[14] M. Hadjinicolaou and D. A. Goussis. "Asymptotic Solution of Stiff PDE's with the CSP Method - The Reaction Diffusion Equation," submitted for publication, SIAM Journal of Scientific Computing, 1996.

[15] U. Maas, S.B. Pope. "Simplifying Chemical Kinetics: Intrinsic Low-Dimensional Manifolds in Composition Space." Combustion and Flame 88:239-264, 1992.

[16] U. Maas, S.B. Pope. "Implementation of Simplified Chemical Kinetics Based On Intrinsic Low-Dimensional Manifolds," TwentyFourth Symposium (International) on Combustion. The Combustion Institute, 1992, pp. 103112.

[17] U. Maas. "Simplifying Chemical Kinetics Using Intrinsic Low-Dimensional Manifolds." Gas Phase Chemical Reaction Systems, Springer Series in Chemical Physics, Volume 61, J. Wolfrum, R. Rannacher, and J. Warnatz, eds., Springer-Verlag, 1996.

[18] K. K. Kuo. Principles of Combustion, John Wiley \& Sons, 1986.

[19] D. R. Mott. "New Quasi-Steady-State and Partial-Equilibrium Methods for Integrating Chemically Reacting Systems." Ph.D. Thesis, The University of Michigan, April 1999.
[20] Charles D. Hodgman, Editor. C.R.C. Standard Mathematical Tables, 12th edition, The Chemical Rubber Publishing Company, Cleveland, Ohio, 1959.

[21] A. M. Khokhlov, "Stability of detonations in supernova." The Astrophysical Journal, Vol. 419, pp. 200-206 (1993).

[22] D. R. Mott, E. S. Oran, B. van Leer. "A QuasiSteady-State Solver for the Stiff Ordinary Differential Equations of Reaction Kinetics." Journal of Computational Physics 164, pp. 407-428 (2000).

[23] D. R. Mott, E. S. Oran, B. van Leer. "CHEMEQ2: A Solver for the Stiff Ordinary Differential Equations of Chemical Kinetics," NRL Memorandum Report NRL/MR/6400- 01-8553, Naval Research Laboratory, Washington, D.C. (2001).

[24] W. H. Vandevender and K. H. Haskell, "The SLATEC mathematical subroutine library," SIGNUM Newsletter, 17, 3 (September 1982), pp. 16-21. 